This is an open access article distributed under the terms of the Creative Commons BY-NC-ND Licence

\title{
MicroRNA319 family members play an important role in Solanum habrochaites and $S$. lycopersicum responses to chilling and heat stresses
}

\author{
X.P. SHI ${ }^{1,2}$, F.L. JIANG ${ }^{1,2}$, J.Q. WEN ${ }^{1,2}$, S.Y. CUI ${ }^{1,2}$, Y.Z. ZHOU ${ }^{1,2}$, and Z. WU ${ }^{1,2 *}$ \\ College of Horticulture, Nanjing Agricultural University, Nanjing, 210095, P.R. China ${ }^{1}$ \\ Key Laboratory of Horticultural Plant Biology and Germplasm Innovation in East China, Ministry of Agriculture, \\ Nanjing, 210095, P.R.China ${ }^{2}$
}

\begin{abstract}
The microRNA319 (miR319) family is involved in plant development and responses to abiotic stresses. Previous work showed that miR319 responded to chilling stress in the chilling-tolerant wild tomato (Solanum habrochaites L.) genotype LA1777. Here, the precursors of sha-miR319a, b, c, and d were cloned from LA1777 and the putative target genes tosinte branched/cycloidea/proliferating cell factors (TCP3 and TCP29) were validated using 5'-RLM-RACE. Expression patterns revealed a negative correlation of sha-miR319 with TCP3 and TCP29 in LA1777. Four tomato (S. lycopersicum) genotypes with varying sensitivities to chilling and heat stresses were selected to characterize expression patterns of miR319 and target genes under extreme temperatures. The involvement of miR319 in the chilling tolerance of tomato might be mediated by the repression of TCP3 and TCP29 expression. Initial stages of heat stress resulted in the up-regulation of miR $319 \mathrm{a}, \mathrm{b}$, and $\mathrm{d}$ and led to a decrease in TCP3, TCP29, and TCP2 expression, whereas the down-regulation of miR319c in the later stages of heat stress may have been responsible for the subsequent up-regulation of TCP3, TCP29, and TCP2. Cis-elements found in the promoter regions of the miR319 family members indicated a potential role of miR319 in the regulation of stress tolerance and development processes. This study provides insights into the role of miR319-mediated regulatory mechanisms in responses to temperature stress in tomato genotypes.
\end{abstract}

Additional key words: abiotic stresses, target gene expression, TCPs.

\section{Introduction}

MicroRNAs (miRNAs) are a class of small non-coding, single-stranded RNAs of $19-24$ nucleotides (nt) in length that are widely distributed in plants and animals (Bartel 2004). In plants, miRNAs are highly complementary to messenger RNAs (target mRNAs) and suppress the expression of target genes through cleavage or translational repression (Dugas and Bartel 2004). Studies from a diversity of plant species have shown that miRNAs play important roles not only in plant growth and development (Palatnik et al. 2003, Wang et al. 2009, Zhu et al. 2009, 2011), but also in stress responses (Sunkar and Zhu 2004, Sunkar et al. 2006, Liu et al. 2008, Wang et al. 2012, Phookaew et al. 2014, Huang et al. 2018).

One of the most conserved miRNA families in plants is the miR319. Some of the teosinte branched/cycloidea/ proliferating cell factors (TCP) family members have been reported to be the target genes of miR319 (Palatnik et al. 2003, Nag et al. 2009, Wu et al. 2017). Various aspects of miR319-regulated TCPS have been studied in plant growth and development, including regulation of leaf (Nath et al. 2003, Palatnik et al. 2003, Koyama et al. 2007, Schommer et al. 2008, Koyama et al. 2010, Xie et al. 2017) and flower (Nag et al. 2009) morphogenesis, cell proliferation (Sarvepalli and Nath 2011, Schommer et al. 2014), secondary cell wall biosynthesis (Sun et al. 2017), and phytohormone biosynthesis and signal

Submitted 4 March 2018, last revision 16 July 2018, accepted 1 August 2018.

Abbreviations: CBF - C repeat binding factor; miRNA - microRNA; qPCR - quantitative PCR; 5'-RLM-RACE - 5' RNA ligasemediated rapid amplification of cDNA-end; TCP - tosinte branched/cycloidea/proliferating cell factors.

Acknowledgements: This work was supported by the Priority Academic Program Development of Jiangsu Higher Education Institutions (PAPD), the Foundation Research Project of Jiangsu Province (BK20140712), and the Fundamental Research Funds for the Central Universities, China (KYZ201609).

* Corresponding author: e-mail: zpzx@njau.edu.cn 
transduction (Schommer et al. 2008, Koyama et al. 2010). Furthermore, expression of miR319-mediated target genes has been associated with responses to abiotic and biotic stresses. For example, OsmiR319b impacts leaf morphogenesis and leads to enhanced salinity and drought tolerance by controlling the expression of $P C F 5$, PCF6, and PCF7, and TCP21 (Yang et al. 2013, Zhou et al. 2013), and miR319 may indirectly affect active oxygen scavenging by targeting OsPCF6 and OsTCP21 to regulate plant chilling tolerance (Wang et al. 2014). Evidence of miR319a regulation of GAMYB genes in response to chilling stress has previously been found in tomato plants (Valiollahi et al. 2014), while, heat stress has been shown to cause an increase in the expression of miR319 in radish (Wang et al. 2015) and miR319b has been found to negatively regulate the responses of tomato to root-knot nematode (Zhao et al. 2015).

Solanum lycopersicum is an economically important crop that suffers damage induced by chilling and heat, however, the $S$. habrochaites has been identified as chilling tolerant (Venema et al. 1999, Foolad and Lin 2000, Cao et al. 2015). A large number of miRNAs has been identified in tomato (Cao et al. 2014, Zhao et al. 2015, Omidvar et al. 2015, Zhou et al. 2016), but mechanisms of expression, function, and regulation under temperature stress conditions are poorly understood. Currently, four members of the miR319 family in $S$. lycopersicum have been identified and listed in miRBase 21.0 (http://www.mirbase.org/), comprising miR319a, miR319b, miR319c-3p (the primary form of miR319c, referred to as miR319c hereinafter), and miR319c-5p, while miR319d (initially named shamiR319b stu) has been found in S. habrochaites (Cao et al. 2014, Fan et al. 2015), and S. lycopersicum (Gu et al. 2014). However, little is known about other miR319 family members in $S$. habrochaites.

There is increasing evidence of miR319-mediated responses to chilling and heat stresses (Chen et al. 2012, Wang et al. 2015, Zhou et al. 2016), but the expression mechanisms and target gene responses are unclear, as previous studies have tended to focus on the stress responses in just one member of the miR319 family (Zhou et al. 2010, 2013, Valiollahi et al. 2014). Here, we identified target genes, expression patterns, and upstream promoter regions of members of miR319 in $S$. habrochaites and S. lycopersicum under chilling and heat stresses to provide insights into the role of miR319 in the regulation of temperature stress tolerance in tomato.

\section{Materials and methods}

Plants and treatments: We used Solanum habrochaites L. genotype LA1777 and four Solanum. lycopersicum L. genotypes with varying sensitivities to chilling and heat stresses (LA2683, Pole red Siberian, LA3475, and Stupice; Table 1 Suppl.). Following soaking and pregermination treatments, tomato seeds were sown in plastic plots and raised in growth chambers ( $R D N-560 E-4$ Dongnan Instruments, Ningbo, China) set at day/night temperatures of $25 / 18{ }^{\circ} \mathrm{C}$, an air humidity of $60 \pm 5 \%$, a 14-h photoperiod, and an irradiance of $280 \pm 20 \mu \mathrm{mol}$ $\mathrm{m}^{-2} \mathrm{~s}^{-1}$.

Thirty days after sowing, vigorous and uniform seedlings were selected for the temperature stress treatments, where seedlings of LA1777, LA2683, Pole red Siberian, and LA3475 genotypes were exposed to chilling stress $\left(4{ }^{\circ} \mathrm{C}\right)$ and LA1777, Stupice, Pole red Siberian, and LA2683 genotypes were exposed to heat stress $\left(40{ }^{\circ} \mathrm{C}\right)$ in the growth chambers. Three composite samples of second leaves were collected from five seedlings of each genotype after $0,1,4,8$, and $24 \mathrm{~h}$ of exposure to the chilling and heating treatments. Leaf samples were immediately frozen in liquid nitrogen and stored at $-80^{\circ} \mathrm{C}$ prior to RNA isolation.

Expression of miR319 and target genes in LA1777: Roots, stems, and second fully expanded leaves were collected from five 30-d-old seedlings, along with three replicate samples of five opened flowers. Samples were frozen in liquid nitrogen and stored at $-80{ }^{\circ} \mathrm{C}$ prior to

RNA isolation.

Total RNA was extracted using TRIzol reagent (Bioteke, Beijing, China). For cDNA synthesis, $1 \mu \mathrm{g}$ of total RNA was used for reverse transcription using a PrimeScript reverse transcription (RT) reagent kit (Takara, Dalian, China) according to the manufacturer's recommendations. Stem-loop RT primers for miR319 (Table 2 Suppl.) were designed according to the criteria described by Tang et al. (2006) and were amplified using SYBR Premix Ex Taq (Takara) in a Quantstudio3 realtime PCR system (Applied Biosystems, Foster City, USA) according to the manufacturer's instructions. There were three replicate PCR reactions for each sample.

Real-time quantitative (q)PCR reactions of miRNAs and target genes were performed with an initial cycle at $95^{\circ} \mathrm{C}$ for $30 \mathrm{~s}$, followed by 40 cycles at $95^{\circ} \mathrm{C}$ for $5 \mathrm{~s}$ and at $60{ }^{\circ} \mathrm{C}$ for $30 \mathrm{~s}$. Tomato uracil riched non-coding small nuclear RNA6 (U6snRNA) and Actin were used as reference genes of miRNAs and mRNAs, respectively. The reactions were repeated three times per sample and expressions were calculated using the $2^{-\Delta \Delta \mathrm{C}_{\mathrm{T}}}$ method (Livak and Schmittgen 2001). Data were analyzed using analysis of variance (ANOVA) in SPSS 18.0 and figures were drawn using Origin 7.0.

Cloning miR319d: Total RNA of LA1777, LA2683, Pole red Siberian, LA3475, and Stupice genotypes were transcribed into cDNA using miR319d stem-loop RT primers (Table 2 Suppl.). PCR was performed using 
Premix Taq (Takara) according to the manufacturer's recommendations, and PCR products were cloned into pMD19-T vector (Takara) prior to sequencing.

Three pairs of primers (sly-pre-aF and sly-pre-aR; sly319 bre $1 \mathrm{~F}$ and sly-319bpre2R; and, sly-319cpre3F and sly-319cpre1R) were designed based on miRBase 21.0, to clone the precursors of miR319a, b, and c (MIR319a, b, and c, respectively) in LA1777 genotype. We searched for the possible precursor of miR319d from the Tomato Genome SL3.0 (https://www.solgenomics.net/) using Blast tools; about $200 \mathrm{nt}$ extended sequences were selected for secondary structures and analyzed using RNAfold software (http://rna.tbi.univie.ac.at/cgibin/RNAfold.cgi). Only sequences with a stable hairpin structure were considered as precursors of miR319d (MIR319d). Forward (sha-319-3-F) and reverse (sha-319$3-\mathrm{R})$ primers were designed based on 200 bp up- and down-stream of $5^{\prime}$ and $3^{\prime}$ sequence of MIR319d position, respectively.

Verification of target genes: To verify the target genes, a modified 5' RNA ligase-mediated RACE (5'-RLMRACE) assay was carried out according to the FirstChoice RLM-RACE procedure (Ambion, CA, USA). The nested PCR products were cloned into $p E A S Y$ Blunt Simple cloning vector (Trans, Beijing, China) and sequenced to validate the cleavage sites and confirm the putative target genes.

Approximately $2500 \mathrm{bp}$ upstream precursor sequences of each member of MIR319 were selected as promoter regions and analysis was carried out using the PlantCARE (http://bioinformatics.psb.ugent.be/webtools/ plantcare $/ \mathrm{html})$.

\section{Results}

A sequence comparison showed mismatches between sha-miR319d and the known members of sly-miR319 (Fig. 1A). We isolated, characterized, and cloned miR319d from $S$. habrochaites LA1777 and S. lycopersicum LA2683, Pole red Siberian, LA3475, and Stupice genotypes (Fig. $1 B$ and Fig. $1 A$ Suppl.).

To investigate all members of miR319 in S. habrochaites, we cloned and sequenced 169, 292, and 195 bp DNA fragments, where similarity between sly-MIR319a, sly-MIR319b, and sly-MIR319c was 95.37, 98.36, and $100.00 \%$, respectively (Fig. 1C-E). Therefore, we named the obtained sequences sha-MIR319a, sha-MIR319b, and sha-MIR319c (GenBank accession numbers MH230178, MH230179, and MH230180), respectively. The sequence of miR319d mapped to the tomato genome, where the extended sequence SL3.0ch03 (69596746-69596918) formed a stable hairpin structure, amplified sha-MIR319d (GenBank accession number MH230181) using the LA1777 DNA template (174 bp in length) and shared 98.21 and $95.43 \%$ similarity to stu-MIR319b and sly-MIR319d, respectively (Fig. $1 F$ ). There was a stable hairpin structure in sha-MIR319a, b, c, and d (Fig. 1B-E Suppl.) and the minimum free energy (MFE) was -348.53 , $-479.49,-338.49$, and $-363.17 \mathrm{~kJ} \mathrm{~mol}^{-1}$, respectively. Minimal folding free energy index (MFEI) is an important feature for miRNA identification (Bonnet et al. 2004) and it was $1.01,1.07,0.87$, and 1.06, respectively. MFE of slyMIR319d was $-353.97 \mathrm{~kJ} \mathrm{~mol}^{-1}$ and MFEI was 1.03 (Fig. $1 F$ Suppl.).

Several putative miR319 target genes were predicted using high-throughput degradome sequencing (Cao et al. 2014) and psRNATarget web server (http://plantgrn.noble. org/psRNATarget/) (Dai and Zhao 2011) (Table 3 Suppl.), and we validated the cleavage sites of target genes TCP3 (Solyc12g014140.1.1, International Tomato

Annotation, ITAG v. 2.40) and TCP29 (Solyc08g048370.2.1). Further cloning and sequencing showed the cleavage site was predominantly located between the tenth and eleventh $\mathrm{C}$-nucleotides, and there was correspondence between sequences of miR319 members (Fig. 2). There were two mismatches between corresponding sites of TCP3 and miR319a, miR319c, and miR319d, and between corresponding sites of TCP29 and miR319c and miR319d. There was greater mismatch between TCP3 and miR319b, TCP29 and miR319b, and TCP29 and miR319a. The specific bands of TCP3 and TCP29 were detected at 280 and 230 bp (Fig. 2 Suppl.).

We found that expression of miR319 tended to be greatest in stems of LA1777 and lowest in its leaves. Expression of miR319a was consistently greater than that of the other members in roots, stems, and flowers, and expression of miR319b-d was consistently low in roots, leaves, and flowers (Fig. $3 A$ ). The lowest expressions of TCP3 and TCP29 were in the stems and highest in the flowers, where expression was negatively correlated with that of miR319. There was some variation in pattern of target gene expression, where expression of TCP3 was higher in leaves than in roots, but that of TCP29 was higher in roots (Fig. 3B).

To investigate the role of miR319 in response to chilling, tomato genotypes with varying sensitivities to chilling (Fig. 4A-D) were used to explore expressions of the members of miR319 (Fig. 4E-H). After a 24-h treatment, expressions of all four members were lower in the LA1777 genotype than in the three cultivated tomato genotypes, including the chilling tolerant LA2683, and expressions of miR319c and d were higher after $24 \mathrm{~h}$ than those of miR319a and b. In contrast, we found that transcription of miR $319 \mathrm{~b}$ was higher after $24 \mathrm{~h}$ than those of all other members in each of the three cultivated genotypes (Fig. 4F-H). There were temporal differences 
A

sly-miR319a

sly-miR319b

sly-miR319c-3p

sha-miR319d

stu-miR319b

Consensus

C sly-MIR319a sha-MIR319a

Consensus

sly-MIR319a

sha-MIR319a

Consensus

sly-MIR319a sha-MIR319a

Consensus

sly-MIR319b sha-MIR319b

Consensus

D sly-MIR319b sha-MIR319b

Consensus

sly-MIR319b sha-MIR319b

Consensus

sly-MIR319b sha-MIR319b

Consensus

E sly-MIR319c sha-MIR319c Consensus

sly-MIR319c sha-MIR319c Consensus

sly-MIR319c sha-MIR319c Consensus

F sly-MIR319d sha-MIR319d stu-MIR319b Consensus

sly-MIR319d sha-MIR319d stu-MIR319b Consensus

sly-MIR319d sha-MIR319d stu-MIR319b Consensus
B

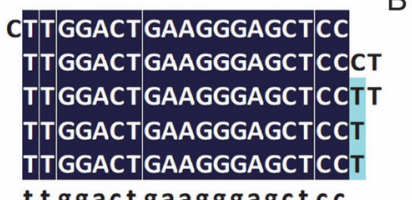

t t ggact gaagggagct c c

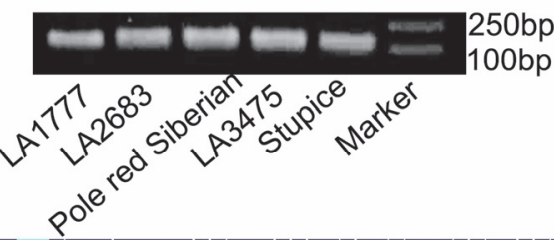

GAGCTT. CTTTTAGTCCACACATGGGGAACGAT/AGGGTTCAATTTIGCTIGCCGACTICATTCA GAGCTITCTITCGGTCCACACATGGGGGGCGATAGGGTTCAATTTGCTGCCGACTCATTCA gagctt ct gtccacacatggg cgatagggttcaatttgctgccgactcattca

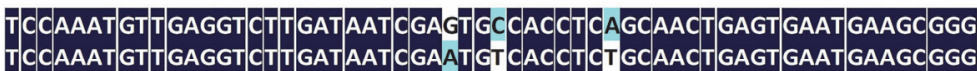

ICCAAAT GT T GAGGT CTI GATAAT CGAAT GT CACCT CT GCAACT GAGT GAAT GAAGCGGC miR319a

AGTACAAGTTIGAGTCTTTGAGTTTCCT GTACTTIGGACT/GAAGGGAGCTIC

AGTACAAGTTIGAGTCTITGAGCTTCCTIGTACTTGGACT GAAGGGAGCTCC

agt acaagt tgagt ct tgagct tcctgtact tggactgaagggagct cc

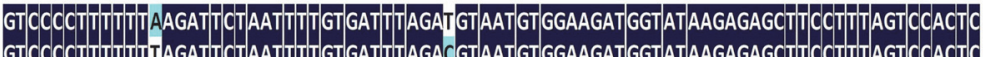

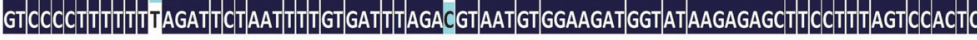
gtccctt tt t agat tctaat ttgtgat ttaga gtaatgtggaagatggt at aagagagct cct ttagtccactc

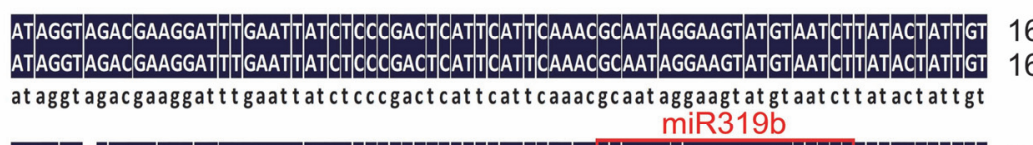

miR319b

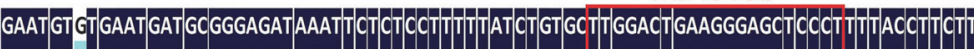

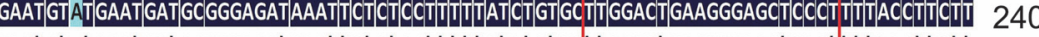
gaat gt $t$ gaat gat gcgggagat aaat $t c t c t c c t t t t$ at ct gt gct t ggact gaagggagct ccct t t t acct tct t

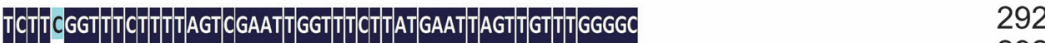

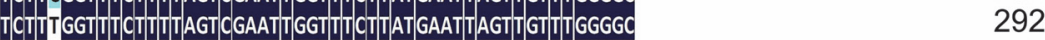

tct t ggt tect tt agt cgaat ggt tt ct t at gaat tagt t gt t t ggggc

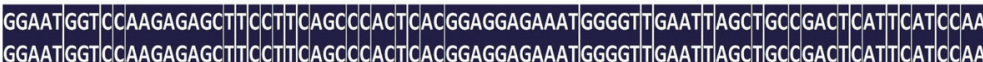

GGAAT GGI CCAAGAGAGCTICCTICAGCCCACTCACGGAGGAGAAAT GGGGTIGAATTAAGCT GCCGACTCATIICATCCAA ggaat ggt ccaagagagct t c ct $t$ cagc c c act c acggaggagaaat ggggt t gaat $t$ agct gc c gact cat t cat c caa

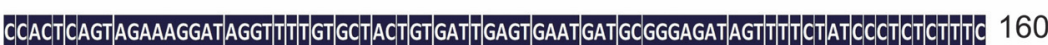

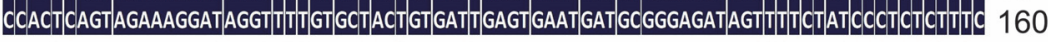
ccact cagt agaaaggat aggt t t t gt gct act gt gat tgagt gaat gat gcgggagat agt t t ct at ccct ctct t $\mathrm{c}$ $\operatorname{miR} 319 \mathrm{c}$

$$
\begin{aligned}
& 195 \\
& 195
\end{aligned}
$$$$
\text { CTTGCTIGGACTIGAAGGGAGCTCCTTTTICCCCATT }
$$$$
\text { ct tgct tggactgaagggagctccttt tccccat }
$$

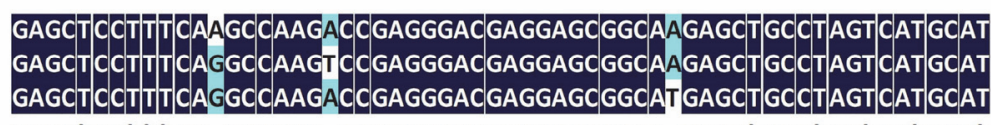
gagctccttca gccaag ccgagggacgaggagcggca gagctgcctagtcatgcat

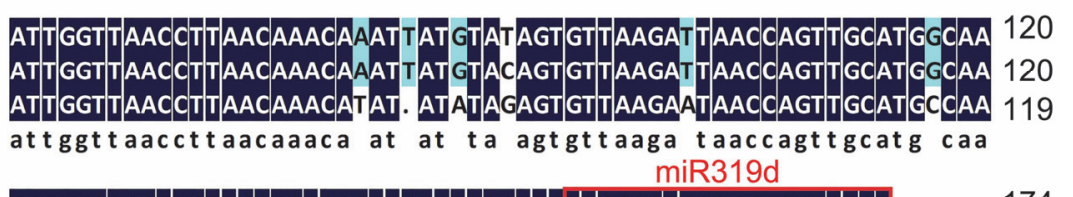

GGGAGCAACT/ACTTICCGCTAACTTIGTCTCACTTIATTGGACTIGAAGGGAGCTTCCT

gggagcaact act t c gct aact tgtctcact t at tggactgaagggagctcct

Fig. 1. Cloning and sequence analysis of miR319d and sha-MIR319. A - The sequence similarity alignment between sly-miR319, sha-miR319d and S. tuberosum stu-miR319b. B - Electrophoresis of stem-loop reverse transcription PCR products of miR319d in different tomato genotypes. $C$ - Sequence comparison of sha-MIR319a and sly-MIR319a. $D$ - Sequence comparison of sha-MIR319b and sly-MIR319b. E - Sequence comparison of sha-MIR319c and sly-MIR319c. F - Sequence comparison of sha-MIR319d, sly-MIR319d (tomato genome SL3.0ch03:69596746-69596918) and stu-MIR319b. The dark blue colour indicates the same bases and the light blue colour indicates different bases, respectively. Red rectangle indicates mature miR319 regions. 
in rates of expression of the members of miR319 among the cultivated genotypes of varied cold tolerance, where miR319b increased more rapidly and to a higher level in the chilling-tolerant LA2683, whereas miR319a expressions was higher in the chilling sensitive genotype LA3475 than in the more chilling tolerant genotypes within $8 \mathrm{~h}$ of treatment (Fig. $4 F-H$ ).

Expressions of TCP3 and TCP29 were lower after chilling in all four tomato genotypes (Fig. 4I-L). TCP2 expression in the wild genotype LA1777 was inhibited during chilling (Fig. 4I), whereas in the cultivated genotypes LA2683, Pole red Siberian, and LA3475, there was a temporal increase (Fig. 4G-L).

We found visible effects of heat stress in all four genotypes, albeit reduced in the heat-tolerant Stupice (Fig. 5A-D) and these were reflected in expressions of

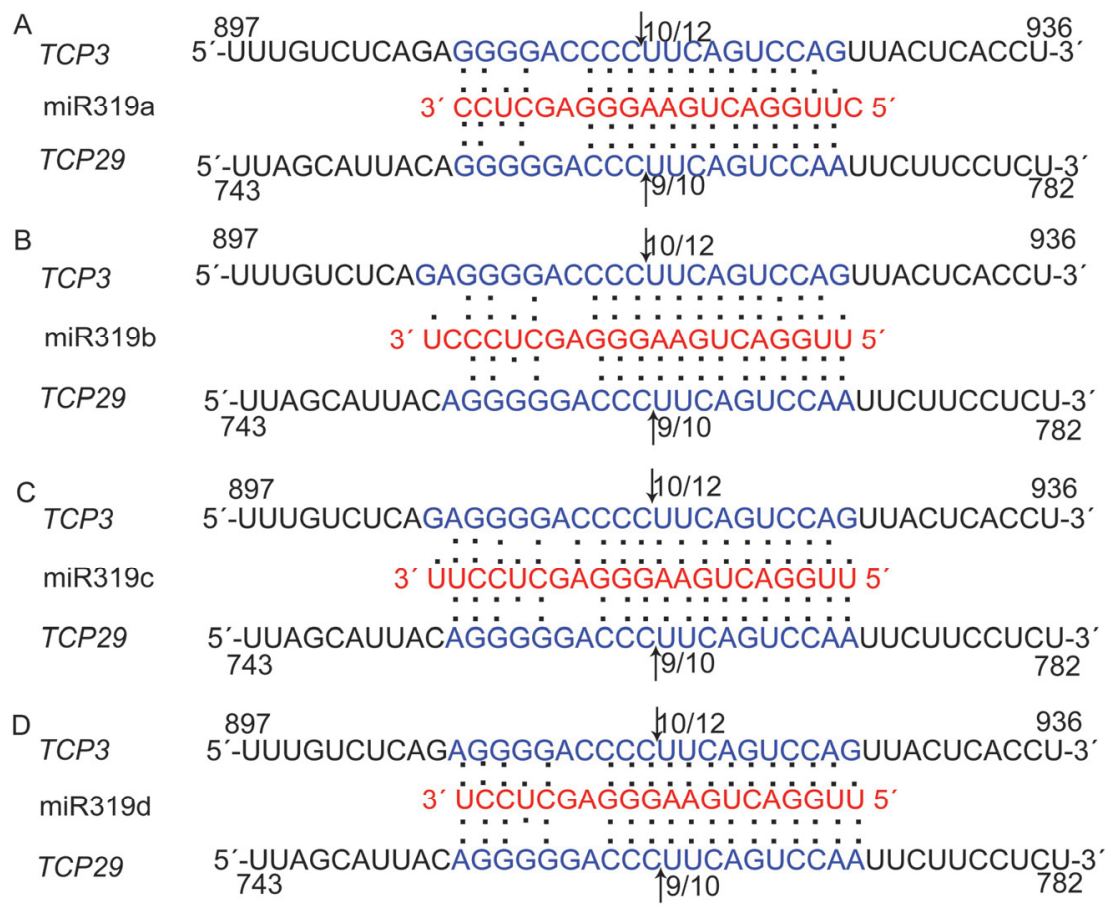

Fig. 2. The miR319 guided cleavage and the target sites validated by $5^{\prime}$ RACE analysis. $A$ - Corresponding sites of $T C P 3$ and $T C P 29$ to miR319a. $B$ - Corresponding sites of TCP3 and TCP29 to miR319b. $C$ - Corresponding sites of TCP and TCP29 to miR319c. $D$ - The corresponding sites of $T C P$ and, $T C P 29$ to miR319d. The numbers indicate the fraction of cloned PCR products terminating at the position.

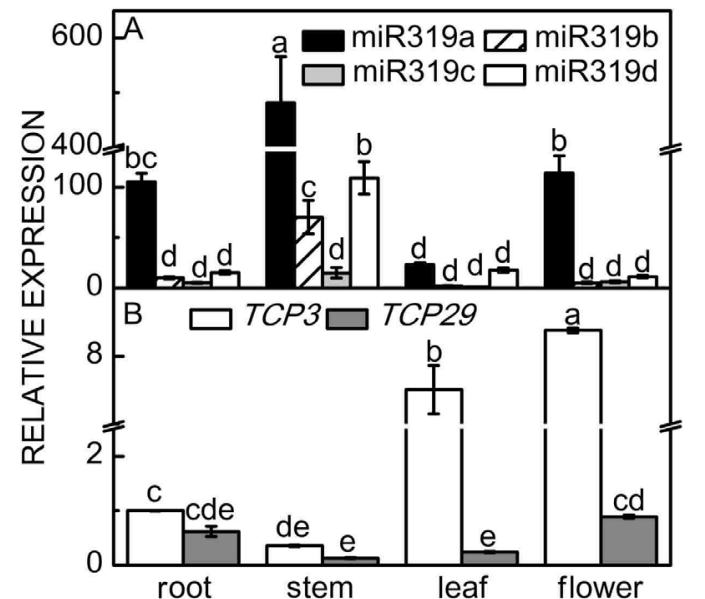

Fig. 3. The qPCR analysis of the expressions of miR319 $(A)$ and target genes $(B)$ in LA1777 genotype. The reference gene for miRNAs was U6snRNA and for target genes was Actin. Means \pm SEs, $n=3$. Different letters indicated significant difference $(P \leq 0.05)$.
miR319 (Fig. 5E-H). Expressions of miR319c decreased from 1 to $8 \mathrm{~h}$ in response to heat in all genotypes (Fig. 5E-H).

Target gene expressions were consistently lower in LA1777 than in the cultivated genotypes following heat treatment (Fig. 5I-L) and the expressions of TCP3 and TCP29 were inhibited following heat treatment in the wild genotype (Fig. 5I). Heat triggered a great decrease in the expression of TCP3 within $1 \mathrm{~h}$ of treatment in the four genotypes (Fig. 5I-L). We also detected a large decrease in the expression of TCP29 within $1 \mathrm{~h}$ of treatment in the cultivated genotypes, except in the heatsensitive LA2683 (Fig. 5J-L). Expressions of TCP2 initially decreased and then peaked after $8 \mathrm{~h}$ of exposure to heat (Fig. 5I-L). We analyzed the temporal expression patterns of miR319 members and target genes, and found a negative correlation between expression of miR319a, $b$, and $\mathrm{d}$ and the target genes after the first hour of heat treatment (Fig. 3A-D Suppl). There was a negative correlation between increased expressions of miR319a, b, 
and $\mathrm{d}$ and the target genes up to $8 \mathrm{~h}$ of treatment in the cultivated genotypes (Fig. $3 E-F$ Suppl.). In order to elucidate miR319 family function in a stress response, the $2500 \mathrm{bp}$ sequence upstream of each member of
miR319 was analyzed. A number of cis-acting, stress related elements were identified from each of the four members of miR319 where expression of miR319b was associated with the greatest number of elements (Table 1).
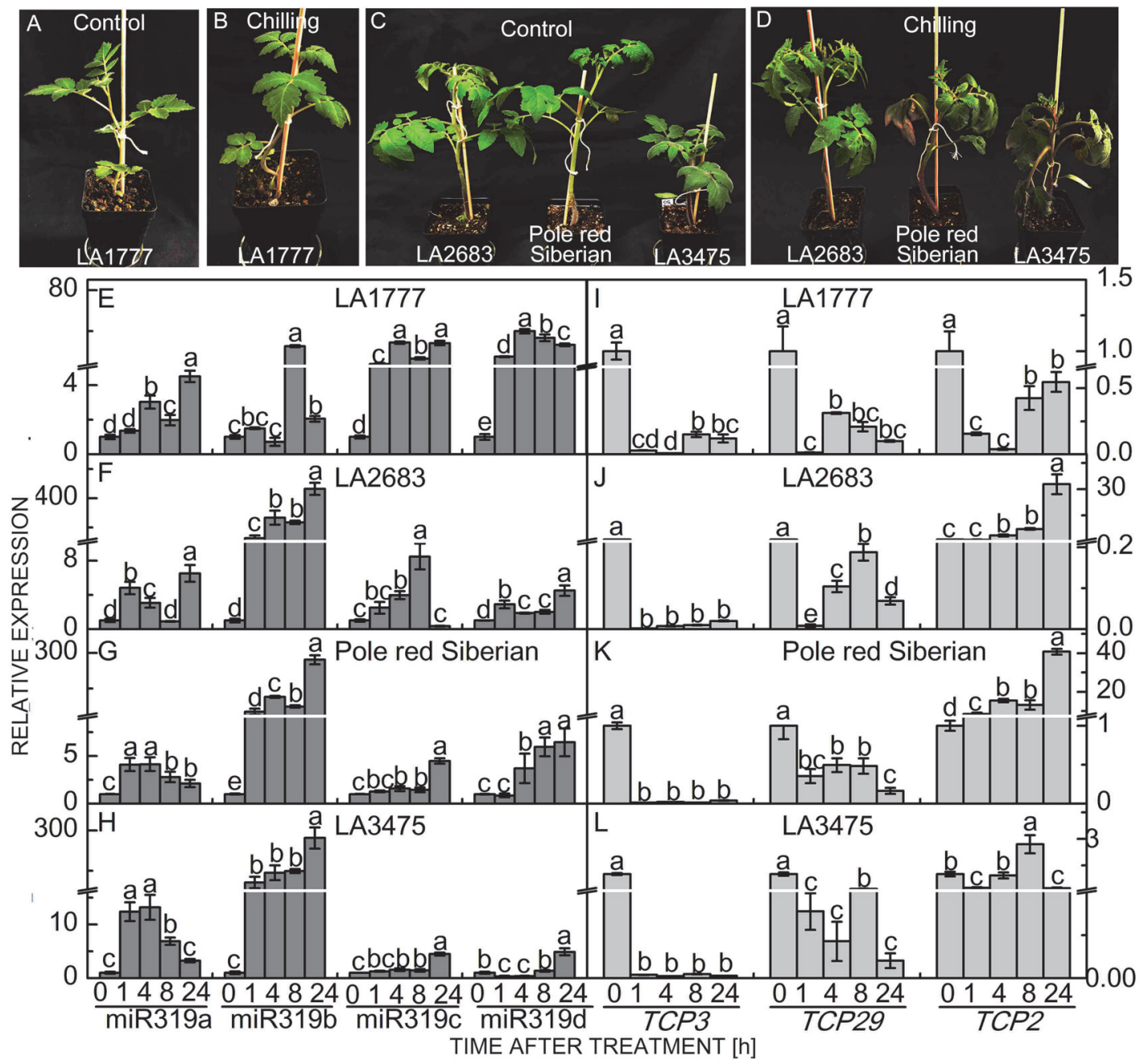

Fig. 4. Different tomato genotypes $(A-D)$ and relative expressions of miR319 $(E-H)$ and target genes $(I-L)$ in these genotypes under chilling stress $\left(4^{\circ} \mathrm{C}\right.$ for $\left.0-24 \mathrm{~h}\right)$. Reference genes for miRNAs and target genes were U6snRNA and Actin, respectively. Means \pm SEs, $n=3$. Different letters indicate significant differences $(P \leq 0.05)$.

\section{Discussion}

We used three key methods to identify miRNAs and, based on the conserved sequence and secondary structures in S. lycopersicum, we cloned sha-MIR319a, sha-MIR319b, and sha-MIR319c from S. habrochaites genotype LA1777 and used bioinformatics prediction to clone sha-MIR319d. High sensitivity, stem-loop RT PCR has been used previously to identify miRNAs (VarkonyiGasic et al. 2007, Li et al. 2009) and using this technique, we cloned miR319d in the wild genotype $S$. habrochaites and S. lycopersicum genotypes to confirm miR319d as a new member of miR319 in cultivated tomato. We found that $5^{\prime}$ fragments of cleaved TCP3 and TCP29 enriched the miR319 target members in the tomato genotypes.

We explored expression patterns of miR319 and target genes under chilling and heat stresses with the aim of identifying the mechanism of miR319-regulated temperature stress tolerance in tomato. We found that expression of all four miR319 members increased as soon as $1 \mathrm{~h}$ after chilling in the chill-tolerant tomato genotypes LA1777 and LA2683, whereas expression of miR319c 
and miR319d increased later in moderately chill-tolerant genotype Pole red Siberian and chill-sensitive genotype LA3475. These results indicated a positive correlation between the expression of miR319 members and chilling tolerance in tomato, because more rapidly elevated expression of miR319 led to improvement in tolerance. We found that the increase in miR319d was greater in
LA1777, while that of miR319b was greater in the cultivated $S$. lycopersicum genotypes. When we assessed heat stress, we found a similar trend of miR319 expression in the genotypes, where was the up-regulation of miR319a, b, and d and down-regulation of miR319c. This also indicated the variability in response by different members of miR319 to heat stress.
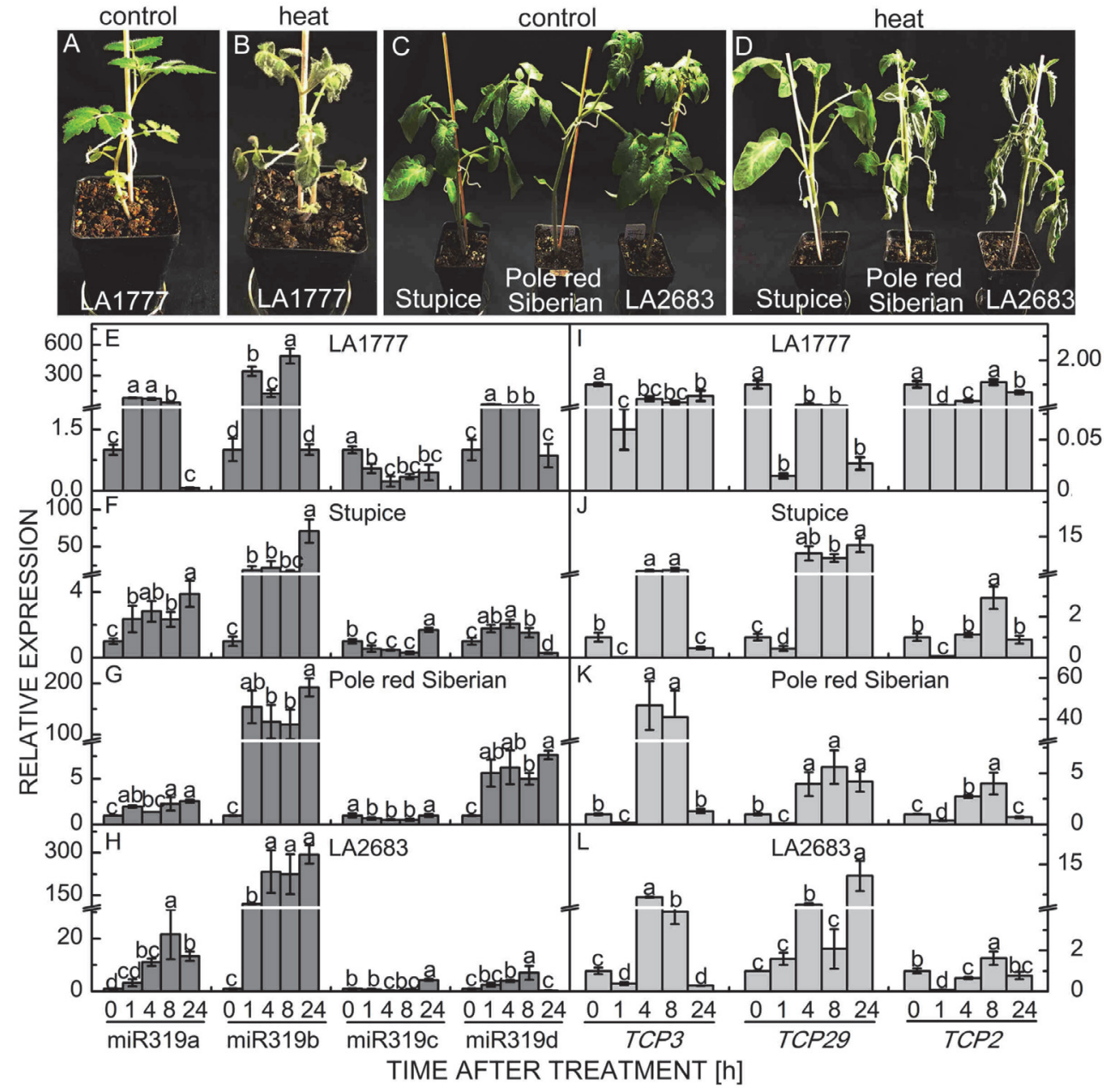

Fig. 5. Different tomato genotypes $(A-D)$ and relative expression levels of miR319 $(E-H)$ and target genes $(I-L)$ in these genotypes under heat stress $\left(40^{\circ} \mathrm{C}\right.$ for $\left.1-24 \mathrm{~h}\right)$. Reference genes for miRNAs and target genes were U6snRNA and Actin, respectively. Means \pm SEs, $n=3$. Different letters indicate significant differences $(P \leq 0.05)$.

Generally, target mRNA expression is negatively correlated with miRNA expression. However, expression patterns of miRNA and target genes may be complex due to variable environmental conditions, as it was shown for miR408 and its targets by Ma et al. (2015). Also, a complementary relation between miR393 and target AFB1 has not been found in Arabidopsis (Chen et al. 2011). In this study, the expressions of TCP3 and TCP29 were lower under chilling stress, whereas those of $T C P 2$, which is a miR319 target in tomato, were not negatively related to miR319. This illustrated that miR319 regulated chilling tolerance through the inhibition of TCP3 and $T C P 29$, but not $T C P 2$. For heat stress, where there were negative correlations between miR319a, $\mathrm{b}$, and $\mathrm{d}$ and the target genes TCP $3, T C P 29$, and TCP2 after $1 \mathrm{~h}$ of treatment, and a negative correlation between decrease in miR319c after 4 and $8 \mathrm{~h}$ of treatment and of expression of $T C P 3, T C P 29$, and TCP2. Thus, there was regulation of $T C P 3, T C P 29$, and TCP2 by miR319a, b, and $\mathrm{d}$ at the initial stage of heat stress, whereas longer term regulation of the target genes was mediated by miR319c.

To further explore the roles of TCP3 and TCP29 under chilling and heat stresses, we identified their functional interactions using STRING software (https://string-db.org/) (Fig. 4 Suppl.). Calcineurin B-like interacting protein kinases 6 (CIPK6) has been predicted 
to interact with TCP3 and a recent study demonstrated that tomato CIPK6 regulates the role of $\mathrm{Ca}^{2+}$ in reactive oxygen species (ROS) signaling pathways (GutiérrezBeltrán et al. 2017). ANT2 (involved in anthocyanin synthesis) and chilling and heat responsive GAMYB-like proteins (Wang et al. 2012, Valiollahi et al. 2014) are predicted to interact with TCP29. Anthocyanins may serve as antioxidants and regulate stress resistance (Zhang et al. 2010, Meng et al. 2014) and AtTCP3 is involved in anthocyanin synthesis in Arabidopsis ( $\mathrm{Li}$ and Zachgo 2013). In this study, we found MBSI, which is the MYB binding site involved in flavonoid biosynthetic gene regulation, in the miR319a and miR319b promoter regions, suggesting the involvement of miR319 with $T C P 3$ and TCP29 in temperature stress regulation as a result of ROS and $\mathrm{Ca}^{2+}$ signaling and synthesis of anthocyanins. However, these suggested mechanisms of TCP3- and TCP29-mediated temperature stress regulation require confirmation.

Promoters contain various functional elements that control the initiation of gene transcription by activating
RNA polymerase (Mitsuhara et al. 1996). For example, the $\mathrm{C}$-repeat binding factors (CBFs) bind to the C-repeat (CRT) and dehydration-responsive element (DRE) in the promoter of cold responsive genes (COR); the plant specific transcription factor WRKY binds to W-box, to regulate responses to cold, heat, salinity, drought, oxidative stress, salicylic acid, abscisic acid (ABA), and ethylene (Jiang et al. 2017); and, anaerobic induction elements (AREs) participate in hypoxic, low-temperature, and dehydration stress responses (Dolferus et al. 1994, Liu et al. 2008). In our study, heat shock response element (HSE) was found in the promoter region of all miR319 members and it may account for the direct induction of miR319a, b, and $d$, and the inhibition of miR319c by heat stress. We identified other, nontemperature stress response elements (Table 1) that indicated the participation of miR319 in response to chilling stress via various signal transduction pathways, while elements related to gibberellic acid, auxin, ethylene and ABA implied a potential regulatory role of miR319 in plant development processes.

Table 1. Types and number of known stress-related elements in the upstream regions of miR319 in S. lycopersicum. For abbreviations see text.

\begin{tabular}{|c|c|c|c|c|c|}
\hline Element & Function & $\operatorname{miR} 319 a$ & $\operatorname{miR} 319 b$ & $\operatorname{miR} 319 c$ & $\operatorname{miR} 319 d$ \\
\hline ARE & stress & 1 & 1 & 2 & 1 \\
\hline MBS & drought stress & 1 & & 2 & 2 \\
\hline HSE & heat stress & 3 & 2 & 3 & 2 \\
\hline TC-rich repeats & defense and stress & 1 & 4 & 1 & 1 \\
\hline Box-W1 & fungal elicitor & 1 & 2 & 2 & 1 \\
\hline AuxRR-core & auxin & 1 & & & \\
\hline TGA-element & auxin & & 1 & 1 & \\
\hline CGTCA-motif & methyl jasmonate & 1 & 1 & 1 & 3 \\
\hline TGACG-motif & methyl jasmonate & 1 & 1 & 1 & 3 \\
\hline GARE-motif & gibberellic acid & 1 & & & \\
\hline TATC-box & gibberellic acid & & & 1 & \\
\hline ERE & ethylene & 2 & 8 & 2 & 1 \\
\hline ABRE & abscisic acid & 1 & & 4 & \\
\hline TCA-element & salicylic acid & & 1 & 4 & \\
\hline MBSI & flavonoid biosynthesis regulation & 1 & 1 & & \\
\hline W box & WRKY binding site & 1 & 2 & 1 & 1 \\
\hline
\end{tabular}

Chilling and heat stress responses in plants are complex processes, involving multiple interactions among genes. We propose a hypothetical model of miR319 regulation of temperature stress in tomato plants (Fig. 5 Suppl.) in which exposure of tomato to chilling induces expression of miR319 that, in turn, inhibits expression of target gene TCPs; in contrast, exposure to heat induces expression of miR319a, b, and $d$, but decreases expression of miR319c that regulates expression of TCPs. We suggest that changes in expression of TCPs in tomato plants may indirectly affect ROS and $\mathrm{Ca}^{2+}$ signaling and anthocyanin synthesis through interactions with CIPK6, GAMYB-like proteins, and ANT2, resulting in enhanced chilling or heat tolerance. Detailed mechanisms of TCPS in chilling or heat stress remain to be determined.

In conclusion, we cloned miR319a, b, c, and d from the wild tomato ( $S$. habrochaites) genotype LA1777 and found a negative correlation between expression of miR319 family members and TCP3 and TCP29 in different plant parts. Up-regulated miR319 family members, which led to repressed TCP3 and TCP29, might promote tolerance to chilling in cultivated tomato genotypes. Expression of miR319a, b, and d increased with heat exposure and inhibited expression of TCP3, $T C P 29$, and $T C P 2$ at the early stage of heat stress, while a 
negative response of miR319c to heat led to increased expression of $T C P 3, T C P 29$, and $T C P 2$ at the later stage of heat stress. The analysis of cis-elements in the promoter regions of miR319 members provided further basis for the study of tomato miR319 family members under various abiotic stresses and plant development.

\section{References}

Bartel, D.P.: MicroRNAs: genomics, biogenesis, mechanism, and function. - Cell 116: 281-297, 2004.

Bonnet, E., Wuyts, J., Rouzé, P., Van de Peer, Y.: Evidence that microRNA precursors, unlike other non-coding RNAs, have lower folding free energies than random sequences. Bioinformatics 20: 2911-2917, 2004.

Cao, X., Jiang, F.L., Wang, X., Zang, Y.W., Wu, Z.: Comprehensive evaluation and screening for chilling tolerance in tomato lines at the seedling stage. - Euphytica 205: 569-584, 2015.

Cao, X., Wu, Z., Jiang, F.L., Zhou, R., Yang, Z.E.: Identification of chilling stress-responsive tomato microRNAs and their target genes by high-throughput sequencing and degradome analysis. - BMC Genomics 15: 1130,2014

Chen, L., Zhang, Y.Y., Ren, Y.Y., Xu, J.C., Zhang, Z.Y., Wang, Y.W.: Genome-wide identification of coldresponsive and new microRNAs in Populus tomentosa by high-throughput sequencing. - Biochem. biophys. Res. Commun. 427: 892-896, 2012.

Chen, Z.H., Bao, M.L., Sun, Y.Z., Yang, Y.J., Xu, X.H., Wang, J.H., Han, N., Bian, H.W., Zhu, M.Y.: Regulation of auxin response by miR393-targeted transport inhibitor response protein 1 is involved in normal development in Arabidopsis. - Plant mol. Biol. 77: 619-629, 2011.

Dai, X.B., Zhao, P.X.C.: psRNATarget: a plant small RNA target analysis server. - Nucl. Acids Res. 39: 155-159, 2011.

Dolferus, R., Jacobs, M., Peacock, W.J., Dennis, E.S. Differential interactions of promoter elements in stress responses of the Arabidopsis Adh gene. - Plant Physiol. 105: 1075-1087, 1994.

Dugas, D.V., Bartel, B.: MicroRNA regulation of gene expression in plants. - Curr. Opin. Plant Biol. 7: 512-520, 2004.

Fan, S.S., Li, Q.N., Guo, G.J., Gao, J.C., Wang, X.X., Guo, Y.M., John, C.S., Du, Y.C.: Identification of microRNAs in two species of tomato, Solanum lycopersicum and Solanum habrochaites, by deep sequencing. - J. integr. Agr. 14: 4249, 2015

Foolad, M.R., Lin, G.Y.: Relationship between cold tolerance during seed germination and vegetative growth in tomato: germplasm evaluation. - J. amer. Soc. hort. Sci. 125: 679$683,2000$.

Gu, M., Liu, W., Meng, Q., Zhang, W.Q., Chen, A.Q., Sun, S.B., $\mathrm{Xu}, \mathrm{G} . \mathrm{H}$.: Identification of microRNAs in six Solanaceous plants and their potential link with phosphate and mycorrhizal signaling. - J. integr. Plant Biol. 56: 11641178,2014

Gutiérrez-Beltrán, E., Personat, J.M., De la Torre, F., Del Pozo, O.: A universal stress protein involved in oxidative stress is a phosphorylation target for protein kinase CIPK6. - Plant Physiol. 17: 836-852, 2017.

Huang, S.C., Lu, G.H., Tang, C.Y., Ji, Y.J., Tan, G.S., Hu, D.Q., Cheng, J., Wang, G.H., Qi, J.L., Yang, Y.H.: Identification and comparative analysis of aluminum-

induced microRNAs conferring plant tolerance to aluminum stress in soybean. - Biol. Plant. 62: 97-108, 2018.

Jiang, J.J., Ma, S.H., Ye, N.H., Jiang, M., Cao, J.S., Zhang, J.H.: WRKY transcription factors in plant responses to stresses. - J. integr. Plant Biol. 59: 86-101, 2017.

Koyama, T., Furutani, M., Tasaka, M., Ohme-Takagi, M.: TCP transcription factors control the morphology of shoot lateral organs via negative regulation of the expression of boundary-specific genes in Arabidopsis. - Plant Cell 19: 473-484, 2007

Koyama, T., Mitsuda, N., Seki, M., Shinozaki, K., OhmeTakagi, M.: TCP transcription factors regulate the activities of ASYMMETRIC LEAVES1 and miR164, as well as the auxin response, during differentiation of leaves in Arabidopsis. - Plant Cell 22: 3574-3588, 2010.

Li, H., Zhang, Z.H., Gao, X.Y., Liu, Y.X., Huang, F.F.: Identification of microRNA in strawberry by RT-PCR. Scientia agr. sin. 42: 1465-1472, 2009.

Li, S.T., Zachgo, S.: TCP3 interacts with R2R3-MYB proteins, promotes flavonoid biosynthesis and negatively regulates the auxin response in Arabidopsis thaliana. - Plant J. 76: 901-913, 2013

Liu, H.H., Tian, X., Li, Y.J., Wu, C.A., Zheng, C.C.: Microarray-based analysis of stress-regulated microRNAs in Arabidopsis thaliana. - RNA 14: 836-843, 2008.

Livak, K.J., Schmittgen, T.D.: Analysis of relative gene expression data using real-time quantitative PCR and the 2(-Delta Delta C (T)) method. - Methods 25: 402 408, 2001

Ma, C., Burd, S., Lers, A.: miR408 is involved in abiotic stress responses in Arabidopsis. - Plant J. 84: 69-187, 2015.

Meng, X., Yin, B., Feng, H.L., Zhang, S., Liang, X.Q., Meng, Q.W.: Overexpression of $R 2 R 3-M Y B$ gene leads to accumulation of anthocyanin and enhanced resistance to chilling and oxidative stress. - Biol. Plant. 58: 121-130, 2014.

Mitsuhara, I., Ugaki, M., Hirochika, H., Ohshima, M., Murakami, T., Gotoh, Y., Katayose, Y., Nakamura, S., Honkura, R., Nishimiya, S., Ueno, K., Mochizuki, A., Tanimoto, H., Tsugawa, H., Otsuki, Y., Ohashi, Y.: Efficient promoter cassettes for enhanced expression of foreign genes in dicotyledonous and monocotyledonous plants. - Plant Cell Physiol. 37: 49-59, 1996.

Nag, A., King, S., Jack, T.: miR319a targeting of TCP4 is critical for petal growth and development in Arabidopsis. Proc. nat. Acad. Sci. USA 106: 22534-22539, 2009.

Nath, U., Crawford, B.C., Carpenter, R., Coen, E.: Genetic control of surface curvature. - Science 299: 1404-1407, 2003.

Omidvar, V., Mohorianu, I., Dalmay, T., Fellner, M.: MicroRNA regulation of abiotic stress response in $7 B-1$ male-sterile tomato mutant. - Plant Genome 8: 3, 2015.

Palatnik, J.F., Allen, E., Wu, X., Schommer, C., Schwab, R.: Control of leaf morphogenesis by microRNAs. - Nature 
425: 257-263, 2003.

Phookaew, P., Netrphan, S., Sojikul, P., Narangajavana, J.: Involvement of miR164- and miR167-mediated target gene expressions in responses to water deficit in cassava. - Biol. Plant. 58: 469-478, 2014.

Sarvepalli, K., Nath, U.: Hyper-activation of the TCP4 transcription factor in Arabidopsis thaliana accelerates multiple aspects of plant maturation. - Plant J. 67: 595-607, 2011.

Schommer, C., Debernardi, J.M., Bresso, E.G., Rodriguez, R.E., Palatnik, J.F.: Repression of cell proliferation by miR319regulated TCP4. - Mol. Plants 7: 1533-1544, 2014.

Schommer, C., Palatnik, J.F., Aggarwal, P., Chetelat, A., Cubas, P., Farmer, E.E., Nath, U., Weigel, D.: Control of jasmonate biosynthesis and senescence by miR319 targets. - PLoS Biol. 6: e230, 2008.

Schwab, R., Palatnik, J.F., Riester, M., Schommer, C., Schmid, M., Weigel, D.: Specific effects of microRNAs on the plant transcriptome. - Dev. Cell 8: 517-527, 2005.

Sun, X.D., Wang, C.D., Xiang, N., Li, X., Yang, S.H., Du, J.C., Yang, Y.P., Yang, Y.Q.: Activation of secondary cell wall biosynthesis by miR319-targeted TCP4 transcription factor. - Plant biotechnol. J. 15: 1284-1294, 2017.

Sunkar, R., Kapoor, A., Zhu, J.K.: Posttranscriptional induction of two $\mathrm{Cu} / \mathrm{Zn}$ superoxide dismutase genes in Arabidopsis is mediated by downregulation of miR398 and important for oxidative stress tolerance. - Plant Cell 18: 2051-2065, 2006.

Sunkar, R., Zhu, J.K.: Novel and stress-regulated microRNAs and other small RNAs from Arabidopsis. - Plant Cell 16: 2001-2019, 2004.

Tang, F., Hajkova, P., Barton, S.C., Lao, K., Surani, M.A.: MicroRNA expression profiling of single whole embryonic stem cells. - Nucl. Acids Res. 34: e9, 2006.

Valiollahi, E., Farsi, M., Kakhki, A.M.: Sly-miR166 and SlymiR319 are components of the cold stress response in Solanum lycopersicum. - Plant Biotechnol. Rep. 8: 349-356, 2014.

Varkonyi-Gasic, E., Wu, R.M., Wood, M., Walton, E.F., Hellens, R.P.: Protocol: a highly sensitive RT-PCR method for detection and quantification of microRNAs. - Plant Methods 3: 12, 2007.

Venema, J.H., Posthumus, F., De Vries, M., Van Hasselt, P.R.: Differential response of domestic and wild Lycopersicon species to chilling under low light: growth, carbohydrate content, photosynthesis and the xanthophyll cycle. Physiol. Plant. 105: 81-88, 1999.

Wang, J.W., Czech, B., Weigel, D.: miR156-regulated SPL transcription factors define an endogenous flowering pathway in Arabidopsis thaliana. - Cell 138: 738-749, 2009.

Wang, R.H., Xu, L., Zhu, X.W., Zhai, L.L., Wang, Y., Yu, R.G., Gong, Y.Q., Limera, C., Liu, L.W.: Transcriptomewide characterization of novel and heat-stress-responsive microRNAs in radish (Raphanus sativus L.) using nextgeneration sequencing. - Plant mol. Biol. Rep. 33: 867-880, 2015.

Wang, S.T., Sun, X.L., Hoshino, Y., Yu, Y., Jia, B., Sun, Z.W., Sun, M.Z., Duan, X.B., Zhu, Y.M.: MicroRNA319 positively regulates cold tolerance by targeting $O S P C F 6$ and
OsTCP21 in rice (Oryza sativa L.). - PLoS ONE 9: e91357, 2014.

Wang, Y., Sun, F.L., Cao, H., Peng, H.R., Ni, Z.F., Sun, Q.X., Yao, Y.Y.: TamiR159 directed wheat TaGAMYB cleavage and its involvement in anther development and heat response. - PLoS ONE 7: e48445, 2012.

Wu, Z.J., Wang, W.L., Zhuang, J.: TCP family genes control leaf development and its responses to hormonal stimuli in tea plant [Camellia sinensis (L.) O. Kuntze]. - Plant Growth Regul. 83: 43-53, 2017.

Xie, Q., Liu, X., Zhang, Y.B., Tang, J.F., Yin, D.D., Fan, B., Zhu, L.H., Han, L.B., Song, G.L., Li, D.Y.: Identification and characterization of microRNA319a and its putative target gene, $P v P C F 5$, in the bioenergy grass switchgrass (Panicum virgatum). - Front. Plant Sci. 8: 396, 2017.

Yang, C.H., Li, D.Y., Mao, D.H., Liu, X., Ji, C.J.: Overexpression of microRNA319 impacts leaf morphogenesis and leads to enhanced cold tolerance in rice (Oryza sativa L.). - Plant Cell Environ. 36: 2207-2218, 2013.

Zhang, K.M., Yu, H.J., Shi, K., Zhou, Y.H., Yu, J.Q., Xia, X.J.: Photoprotective roles of anthocyanins in Begonia semperflorens. - Plant Sci. 179: 202-208, 2010.

Zhao, W.C., Li, Z.L., Fan, J.W., Hu, C.L., Yang, R., Qi, X., Chen, H., Zhao, F.K., Wang, S.H.: Identification of jasmonic acid-associated microRNAs and characterization of the regulatory roles of the miR319/TCP4 module under root-knot nematode stress in tomato. - J. exp. Bot. 66: 46534667,2015

Zhou, L., Liu, Y., Liu, Z., Kong, D., Duan, M., Luo, L.: Genome-wide identification and analysis of droughtresponsive microRNAs in Oryza sativa. - J. exp. Bot. 61: 4157-4168, 2010.

Zhou, M., Li, D.Y., Li, Z.G., Hu, Q., Yang, C.H., Zhu, L.H., Luo, H.: Constitutive expression of a miR319 gene alters plant development and enhances salt and drought tolerance in transgenic creeping bentgrass. - Plant Physiol. 61: 13751391, 2013.

Zhou, R., Wang, Q., Jiang, F.L., Cao, X., Sun, M.T., Liu, M., $\mathrm{Wu}, \mathrm{Z}$.: Identification of miRNAs and their targets in wild tomato at moderately and acutely elevated temperatures by high-throughput sequencing and degradome analysis. - Sci. Rep. 6: 33777, 2016.

Zhou, R., Yu, X.Q., Kjær, K.H., Rosenqvist, E., Ottosen, C., $\mathrm{Wu}, \mathrm{Z}$.: Screening and validation of tomato genotypes under heat stress using $\mathrm{Fv} / \mathrm{Fm}$ to reveal the physiological mechanism of heat tolerance. - Environ. exp. Bot. 118: 1$11,2015$.

Zhu, H., Hu, F., Wang, R., Zhou, X., Sze, S-H., Liou, L.W., Barefoot, A., Dickman, M., Zhang, X.: Arabidopsis argonaute10 specifically sequesters miR $166 / 165$ to regulate shoot apical meristem development. - Cell 145: 242-256, 2011.

Zhu, Q.H., Upadhyaya, N.M., Gubler, F., Helliwell, C.A.: Overexpression of miR172 causes loss of spikelet determinacy and floral organ abnormalities in rice (Oryza sativa). - BMC Plant Biol. 9: 149, 2009. 